\title{
Synergistic Cytotoxic Effects of Ganoderma lucidum and Bacillus Calmette Guérin on Premalignant Urothelial HUC-PC Cells and Its Regulation on Proinflammatory Cytokine Secretion
}

\author{
John Wai-man Yuen, ${ }^{1}$ Mayur-Danny I. Gohel, ${ }^{2}$ and Chi-fai $\mathrm{Ng}^{3}$ \\ ${ }^{1}$ School of Nursing, The Hong Kong Polytechnic University, Hung Hom, Hong Kong \\ ${ }^{2}$ Department of Medical Science, Tung Wah College, Homantin, Hong Kong \\ ${ }^{3}$ Department of Surgery, The Chinese University of Hong Kong, Shatin, Hong Kong
}

Correspondence should be addressed to John Wai-man Yuen, john.yuen@polyu.edu.hk

Received 9 May 2012; Accepted 12 July 2012

Academic Editor: Jae Youl Cho

Copyright (C 2012 John Wai-man Yuen et al. This is an open access article distributed under the Creative Commons Attribution License, which permits unrestricted use, distribution, and reproduction in any medium, provided the original work is properly cited.

Bacillus Calmette-Guérin (BCG) is conventionally used as an adjuvant immunotherapy to reduce the recurrence of bladder cancer. To address the issues of efficacy and safety, an ethanol extract of Ganoderma lucidum (GLe) was evaluated for its interaction with BCG. In a model of premalignant human uroepithelial cells (HUC-PC), GLe exerted immediate cytotoxic effects while BCG showed a delayed response, given that both were immunological active in inducing the secretion of interleukin (IL)-6, IL-8, and monocyte chemotactic protein-1 (MCP-1). Synergistic cytotoxic effects were observed when cells were either coincubated with both drugs or firstly preincubated with GLe. Synergism between GLe and BCG was demonstrated to achieve a complete cytostasis in 24 hours, and such effects were progressed in the subsequent 5 days. However, the pretreatment of GLe resulted in suppression of IL-6, IL-8, and MCP-1 secretions without affecting the cytotoxicity. Given that numerous proinflammatory cytokines are associated with the high side effects toll of BCG, results herein suggested the potential implications of GL to supplement the BCG immunotherapy in bladder cancer, for better efficacy and reducing side effects.

\section{Introduction}

Transitional cell carcinoma (TCC) of the urinary bladder is known for its high recurrence rate up to $80 \%$ if patients are treated by surgical ablation alone [1]. Despite the mechanism of action is not fully understood, Bacillus CalmetteGuérin(BCG) has been used for over 30 years as a prophylactic agent for preventing the TCC recurrence. Intravesical instillation of BCG following transurethral resection (TUR) has shown an overall effectiveness in diminishing $30-40 \%$ of the recurrence and progression [2]. Such effects were relied on the mycobacterium nature of BCG, which was able to trigger local nonspecific immune response through cytokines secretion and recruitment of immune cells to the bladder wall [3]. Several cytokines, some are sourced from urothelial cells, were detected in patients' voided urine upon BCG instillation. In culture experiments, BCG was shown to be active in stimulating tumor-necrosis-factor- (TNF-) related apoptosis in accordance with the production of cytokines including interleukin (IL)-1, IL-6, IL-8, and TNF- $\alpha$, whereas the activity was tumor grade dependent [4]. Besides, BCG also leads to a high side effect toll of $90 \%$ from local cystitis and haematuria, allergic reactions to severe systemic infection [5]. Controversially, the immunological activities induced by BCG, especially some of the cytokines, were suggested as the side effects cause $[6,7]$. Therefore, a new chemopreventive regimen with better efficacy and lesser side effects is demanded.

The recurrence of TCC was associated with the presence of papillary multifocality. This is explained by the "field cancerization hypothesis" and the "clonal seeding theory" that residual adverse cells at premalignant stage could not be completely removed by surgery, and thus they are readily being transformed by any stimulation [1]. In this relation, 
for evaluating potential bladder prophylactic agents, we postulated the importance of utilizing a human uroepithelial (HUC-PC) cell line, which carries the premalignant features of being sensitive to bladder carcinogens for undergoing tumorigenic transformation [8]. Ganoderma lucidum, an ancient medicinal mushroom belonging to the family of Ganodermataceae of Aphyllophorales, has been widely used for promotion of health and longevity. Its popularity and perceived health benefits have also prompted the usage by cancer patients. With polysaccharides and triterpenes as principle bioactive constituents, a range of scientific evidence from in vitro to animals and humans in vivo has been discovered for supporting the antitumorigenicity of $G$. lucidum in cancer of different origins [9]. In the past years, some works have been done to explore the chemopreventive properties of this mushroom on TCC. Antiproliferation was demonstrated on TCC cells with different degrees of malignancy, from premalignant to high-grade invasive [10]. In the premalignant HUC-PC cells, growth inhibition via G2/M phase cell cycle arrest and apoptosis was shown by ethanol extract of G. lucidum (GLe), in addition to the suppression of carcinogen 4-aminobiphenyl-mediated cell migration and telomerase activity $[10,11]$. More recently, GLe was found to be immunologically active to induce secretion of an array of cytokines secretion and neutrophilic migration, in the culture of HUC-PC cells [12]. Such properties generate the next question to ask whether GLe could be synergistic with BCG in elimination of adverse cells of TCC. By using the HUC-PC cell model, a pilot study was conducted and indicated that BCG was noncytotoxic in 24 hours after incubation but stimulated a dose-dependent IL- 6 production via nuclear factor-kappa $\mathrm{B}(\mathrm{NF}-\kappa \mathrm{B})$ pathways. In the present study, interaction between GLe and BCG was evaluated using two treatment approaches: combination therapy and a pretreatment of GLe followed by BCG treatment. Cell viability and expression of cytokines (T-helper (Th)1, Th2 cytokines and chemokines) in response to the treatments were investigated as outcome measures. Furthermore, the cytotoxicities induced by test agents were assessed in a prolonged period, in order to confirm the persistence or delay of response.

\section{Materials and Methods}

2.1. G. lucidum Extract and Chemicals. A proprietary extract consisting of G. lucidum fruiting bodies and cracked spores, branded ReishiMax GLP, was purchased from Pharmanex Inc. (Hong Kong). The active ingredients of the product were standardized to $13.5 \%$ polysaccharides ( $\beta-1,3$-glucans) and $6 \%$ triterpene (ganoderic acids and others), which is the highest extractable levels, whereas the remaining $80 \%$ consisted of nucleosides, fatty acids, and amino acid according to the manufacturer's technical bulletin. The powdered G. lucidum from capsules was re-extracted as previously described [11]. Briefly, capsule contents were dissolved in 95\% HPLC grade ethanol for 30 minutes, and the supernatant was further extracted by successive sonication using absolute ethanol. The reextracts were filtered (through
$0.45 \mu \mathrm{m}$ polypropylene filter) and dried under reduced pressure to yield a water-insoluble extract (GLe) as browncolored powder. For experiments, the GLe was dissolved in absolute ethanol (with final solvent concentration below $0.01 \% \mathrm{vol} / \mathrm{vol}$ ), and then immediately diluted to $200 \mu \mathrm{g} / \mathrm{mL}$ GLe assay media stock with complete media before adding into cultures. Immucyst BCG was sourced from Aventis (Toronto, Canada). The whole vial of attenuated BCG (81 mg dry weight containing 5\% monosodium glutamine) was reconstituted with $3 \mathrm{~mL}$ of the accompanying diluents to make a stock solution containing a minimal $6.6 \times 10^{8}$ colony forming units (CFU). The stock solution was further diluted with complete medium into the working concentrations for culture. The Limulus Amebocyte Lysate (LAL) endpoint chromogenic kit assay (CAPE CO, E. Falmouth, MA, USA) was performed to detect lipopolysaccharides (LPS) contamination in the GLe and BCG. In this assay, Glucashield buffer (CAPE COD) was used to reconstitute pyrochrome to inhibit possible $(1,3)-\beta$-D-glucan present in samples, and thus avoiding potential interference. Aseptic techniques were strictly applied throughout the procedures.

2.2. Cell Culture and Treatment. The HUC-PC cell line (provided by Dr. Rao from the University of California, Los Angeles, USA) was cultured in F12-Ham enhanced Dulbecco's Modified EaGLe's Medium (Sigma, St. Louis, USA) with $1 \%$ penicillin $(10,000 \mu \mathrm{g} / \mathrm{mL})$ and streptomycin $(10 \mathrm{mg} / \mathrm{mL}$ ) and $10 \%$ fetal bovine serum (Gibco Brl Island, New York, USA). All cultures were maintained at $37^{\circ} \mathrm{C}$ in a water-saturated atmosphere containing $5 \% \mathrm{CO}_{2}$. The effects of BCG were tested with or without GLe by using 4 different treatment schedules ( 2 of them were for synergism) illustrated in Figure 1. Logarithmically growing cells were plated in 6-well culture plates at $1.25 \times 10^{5}$ cells per well for treatment $1-3$ and $2.5 \times 10^{5}$ cells per well for treatment 4 . The different cell seeding density in treatment 4 was set to obtain the closest baseline cell numbers as other treatment schedules when corresponding treatment initiated. Treatment 1 and 2 were used to test BCG (at $0,1.2 \times 10^{7}, 2.4 \times 10^{7}$, and $4.8 \times 10^{7}$ $\mathrm{CFU})$ and $G L e(0,40,80,100 \mu \mathrm{g} / \mathrm{mL})$, respectively. Treatment 3 was the combination test for BCG (at fixed concentration of $1.2 \times 10^{7} \mathrm{CFU}$ ) with different concentrations of GLe listed above. Whilst treatment 4 was the GLe-pretreatment protocol that a 24-hour BCG treatment was given following the 24-hour GLe pretreatment. For all treatment schedules, culture media and cells were harvested when the corresponding treatment was completed, and used for measuring the cytokine levels and viable cell count (day 1), respectively. In parallel experiments, cultures were maintained after removal of assay media and pure complete media (in the absence BCG or GLe) was refreshed every 2 days to ensure nutrient supply. On day 6 ( 5 days after retraction of treatment), cells were harvested for viable cell count. Cytokine levels were not determined on day 6 because preliminary data indicated that vast cytotoxicities have appeared in many of the treated cultures that number of viable cells was insufficient for cytokines production. Cells treated with solvent media, that is, $0.1 \% \mathrm{v} / \mathrm{v}$ ethanol for GLe and 33\% v/v diluents for BCG were used as control experiments. 


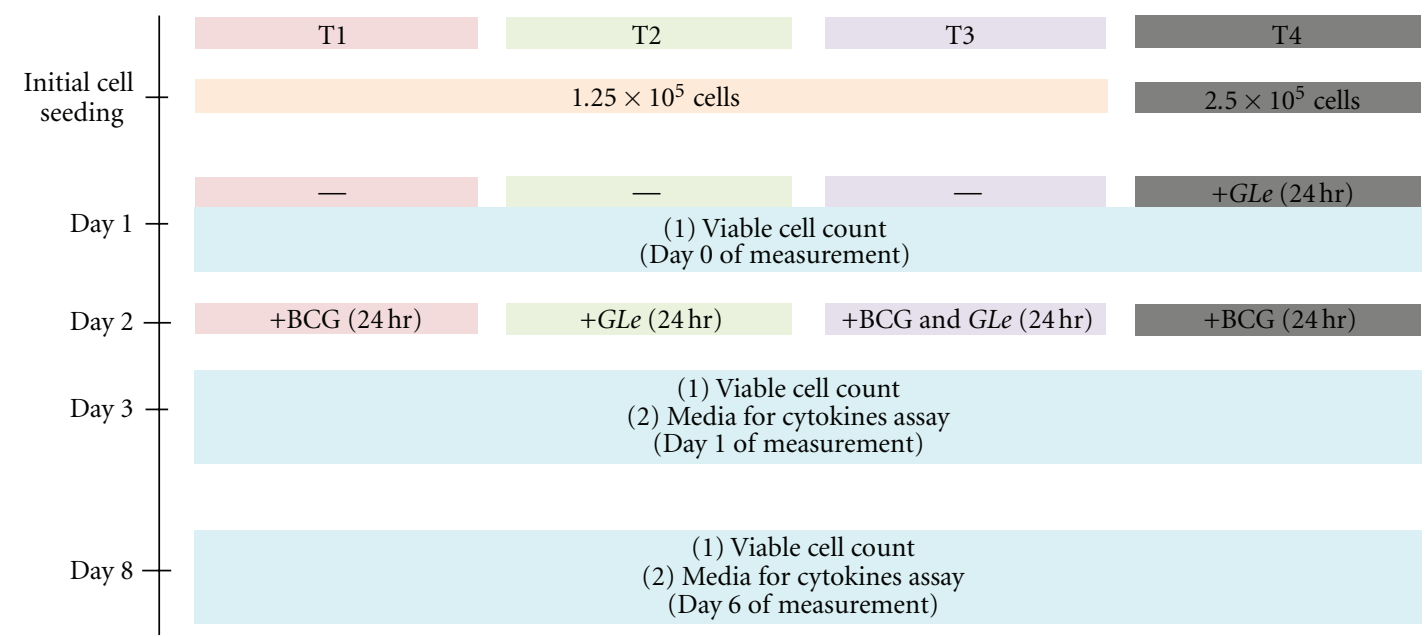

FIGURE 1: The treatment schedules for experiments, illustrating 4 treatments (T1-T4) were tested. A 24-hour incubation was allowed for stabilizing the culture environment after seeding the cells. The initial cell seeding numbers of T1-T3 were halves of T4, because cell cultures for T1-T3 were maintained for an additional 24 hours in complete media, in order to align initial cell numbers for all treatment groups before the first day sampling (Day 0 as baseline), that is, approximately $2.5 \times 10^{5}$ cells since doubling time for the HUC-PC cells was 24 hours. For $\mathrm{T} 4$, a range of GLe concentrations was administrated before the baseline, and this complete medium in the absence of GLe (0 $\mu \mathrm{g} / \mathrm{mL})$ was used as the baseline for this treatment group. Subsequent samples were collected on Day 1 (Day 3 after initial cell seeding) and Day 6 (Day 8 after initial cell seeding) following different treatments.

2.3. Cytotoxicity Assay. Cell viability was assessed by the automated Beckman Coulter Vi-CELL XR cell viability analyzer with its reagent pack (Miami, FL, USA). All cell counting results were verified between the manual and automated methods and expressed as the viable cell numbers for interpretation.

2.4. FlowCytomix for Cytokines Measurement. The FlowCytomix (Bender MedSystems, Austria) human Th1/Th2 11 plex and human chemokine 6plex kits were used for cytokines measurement. In total, 15 cytokines: interferon(IFN)- $\gamma$, IL-1 $\beta$, IL-2, IL-4, IL-5, IL-6, IL-8, IL-10, IL-12p70, tumor necrosis factor (TNF)-, TNF- $\beta$, monocyte chemotactic protein-1 (MCP-1), granulocyte colony stimulating factor (G-CSF), monokine induced by interferon gamma (MIG), macrophage inflammatory protein(MIP)- $1 \alpha$, and MIP-1 $\beta$ were measured, whereas IL- 8 was overlapped in both kits. The Cytomics FC500 Flow cytometer (Beckman Coulter, Miami) equipped with CXP software version 2.2 was used. The forward scatter measurements were collected at 1-8 degrees. Standard mixture was prepared for each kit by mixing the standard of each analyte. The setup beads and standard mixture with highest phycoerythrin (PE) signal (FL-2 585/42 BP for detection) were used to set up the flow cytometry. Dilutions of each standard mixture (total 7 concentrations) were used to create the standard curve. And concentrations of each cytokine in the harvest media were analyzed and read against the corresponding standard curve using the FlowCytomix Pro software (Version 2.2, Bender Medsystem, Austria). Coefficients of variation $<15 \%$ and absence of mavericks were achieved for reliable standard curves.
2.5. Statistical Analysis. All assays were performed in triplicate for reproducibility. Descriptive statistics with mean \pm standard deviation were used to summarize the results. Differences between means were determined using the oneway analysis of variance (ANOVA) followed by Dunnett's test (GraphPad Prism version 3.0 for Windows, San Diego, $\mathrm{Ca}$, USA), whereas statistical significance was sought at twotailed $P$-value of 0.05 .

\section{Results}

3.1. Differentiated Properties of BCG and GLe on HUC$P C$ Cells. Immediately after the 24-hour incubation, no cytotoxicity was demonstrated by BCG, except at $1.2 \times$ $10^{7} \mathrm{CFU}$, about $15 \%$ of viable cell number was reduced (statistically nonsignificant). Nonetheless, a dose-dependent growth inhibition was observed 5 days after the BCG retraction (Figure 2(a)). In contrast, remarkable dose-dependent growth suppression was observed immediately after the 24hour incubation with GLe, whereas the cytostatic effects were further progressed to become more significant in the next 5 days after GLe withdrawal (Figure 2(b)). Consistent with previous findings $[11,13]$, GLe at $80 \mu \mathrm{g} / \mathrm{mL}$ was shown to be growth suppressing that was able to maintain the cell culture at initial seeding cell density for at least 5 days following GLe treatment (as shown in Figure 2(b)) but became cytotoxic to eliminate the adverse cells when concentration reached $100 \mu \mathrm{g} / \mathrm{mL}$ or above.

Cytokine secretion following different treatment schedules were summarized in Table 1. Amongst the 15 cytokines tested, only IL-6, IL-8, and MCP-1 were detectable in the cultures of HUC-PC cells without treatment. BCG (treatment 1) 

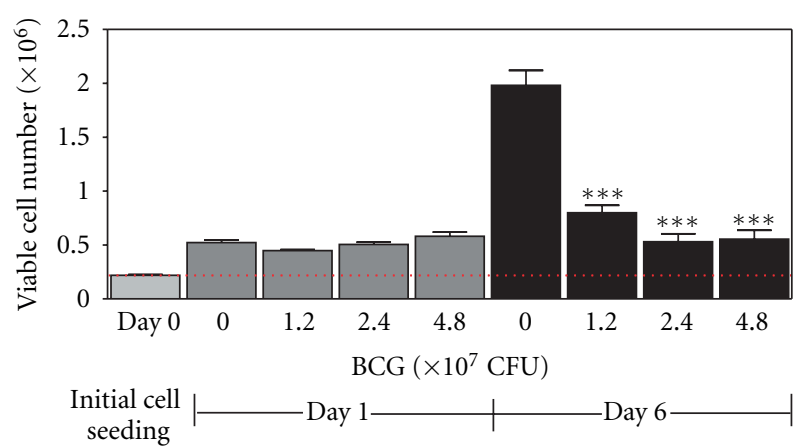

(a)

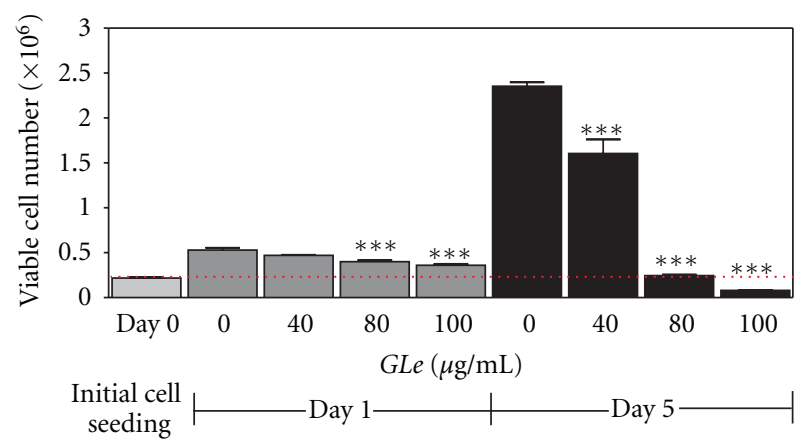

(b)

FIgURE 2: Showing the immediate and progressive cytotoxic effects exhibited by (a) BCG on day $1(F=4.908$; statistically nonsignificant $)$ and day $6(F=51.30 ; * * * P<0.001)$, and (b) GLe on day $1\left(F=18.69 ;{ }^{* *} P<0.001\right)$ and day $6\left(F=118.5\right.$; ${ }^{* *} P<$ 0.001 ). Results of BCG and GLe were statistically compared with the solvent media control according to the corresponding schedule (i.e., day 1 versus Day 1 and Day 6 versus Day 6), at 0 CFU containing BCG diluents $(33 \% \mathrm{v} / \mathrm{v})$ and at $0 \mu \mathrm{g} / \mathrm{mL}$ containing ethanol $(0.1 \%$; $\mathrm{v} / \mathrm{v})$, respectively. All experiments were performed in triplicate for reproducibility. $F$ value of each treatment group was determined by one-way ANOVA, and Dunnett's posttest was followed to determine $P$ values.

and GLe (treatment 2) exhibited similar activities in inducing IL-6 and IL-8 production $(P<0.001)$, reducing MCP-1 levels $(P<0.001$ for all GLe concentrations but $P<0.001$ for BCG at $1.2 \times 10^{7} \mathrm{CFU}$ only). The increase or decrease of cytokines demonstrated by GLe was in clear dose-dependent manner, whereas GLe has also stimulated a trace amount of IL-2 production but statistically nonsignificant. On the contrary, the BCG-mediated cytokines were not dose dependent.

3.2. Synergistic Cytotoxicity between GLe and BCG. The interaction between GLe and BCG on HUC-PC cells was evaluated by another two treatment schedules (treatment 3 and 4 illustrated in Figure 1). Irrespective of combination treatment with GLe (treatment 3) or pretreatment of GLe (treatment 4 ), the growth inhibitory activities induced by $1.2 \times 10^{7} \mathrm{CFU}$ of BCG were significantly enhanced. Cell growth was completely halted (maintaining the initial cell density) and was observed in the 24-hour combination treatment of $80 \mu \mathrm{g} / \mathrm{mL}$ GLe with $1.2 \times 10^{7} \mathrm{CFU}$ BCG (Figure 3(a)). In the following 5 days when the treatment was removed, viable cell numbers were reduced below the initial cell density by all test concentrations, of which $6 \%, 65 \%$, and $80 \%$ of the HUC-PC cells were eliminated by combining $1.2 \times 10^{7} \mathrm{CFU}$ BCG with 40,80 , and $100 \mu \mathrm{g} / \mathrm{mL}$ of GLe, respectively. On the other hand, cytotoxic activities of BCG were remarkably promoted and fastened by the pretreatment with GLe (treatment 4; Figure 3(b)). About $25 \%$ and $40 \%$ of the initial cell densities were eliminated by the pretreatment with 80 and $100 \mu \mathrm{g} / \mathrm{mL}$ of GLe, respectively, immediately after complete cessation of BCG. Such cytotoxic effects were further progressed in the next 5 days, with about $60 \%$ cell reduction by 40 or $80 \mu \mathrm{g} / \mathrm{mL}$ GLe and $73 \%$ cell elimination by $100 \mu \mathrm{g} / \mathrm{mL}$ GLe. To confirm the relationship between GLe and BCG, combination index (CI) values were determined for treatment groups 3 and 4 , where CI $<1$ indicates synergism, $\mathrm{CI}>1$ indicates antagonism, and $\mathrm{CI}=1$ indicates additive effect. Our results (Figure 4(c)) clearly showed that HUC-PC cells treated with BCG showed synergistic loss of cell viability when combined with GLe $(\mathrm{CI}=0.91)$ or pretreated with GLe $(\mathrm{CI}=0.81)$.

3.3. Regulatory Activities of GLe on BCG-Mediated Cytokines. The coincubation of BCG and GLe (treatment 3) has promoted the secretion of IL6 dose-dependently and has diminished the production of IL-8 (statistically nonsignificant) and MCP-1 $(P<0.001)$ (Table 1). Oppositely, all BCG-mediated cytokines (IL-6, IL-8, and MCP-1) were dramatically $(P<0.001)$ suppressed by the 24-hour GLe pretreatment (Table 1). In particularly, MCP-1 level of HUC-PC cells in treatment 4 was dropped to nondetectable level when pretreated with $100 \mu \mathrm{g} / \mathrm{mL}$ of GLe. However, IL-2 was not detectable in all cultures treated under treatment 3 or 4 schedules.

\section{Discussion}

Current in vitro study reports encouraging findings that ethanol extract of Ganoderma lucidum exhibited synergistic cytotoxic effects with BCG on the adverse uroepithelial cells at premalignant stage, in addition to its regulatory effects on proinflammatory cytokines including IL-6, IL-8, and MCP1. Further to our previously reported growth inhibitory properties of GLe, this is the first time to demonstrate that cytotoxicity of GLe or BCG was progressed continuously even after the treatment withdrawal. However, the BCG cytotoxicity exhibited on the HUC-PC cells was shown to be delayed after the BCG cessation.

Previous studies on BCG cytotoxicity were mainly focused on high-grade tumor cells [15], which may not be able to reflect the actual clinical implications in removing the residual cells from the bladder wall. Apoptosis was induced on bladder cancer cell lines of grade 3 and 4 stages by the wall preparation of mycobacterium species [16]. In low-grade bladder tumor cell lines, cell cycle arrests were observed after exposing to BCG, but no DNA degradation was shown, and therefore, apoptotic effects could not be confirmed [17]. Besides, the authors postulated that those residual adverse cells following surgical ablation are premalignant rather than 
TABle 1: Yuen et al.

\begin{tabular}{|c|c|c|c|c|c|}
\hline \multirow{2}{*}{ Treatment schedule } & & \multicolumn{4}{|c|}{ Cytokine level (Mean \pm SD); $n=3$} \\
\hline & & $\mathrm{IL}-2(\mathrm{pg} / \mathrm{mL})$ & IL-6 (pg/mL) & IL-8 (pg/mL) & MCP-1 (pg/mL) \\
\hline \multicolumn{6}{|l|}{ Treatment 1 (24 hours) } \\
\hline \multirow[t]{4}{*}{ BCG alone } & $0 \mathrm{CFU}^{\dagger}$ & N.D. & $76 \pm 21$ & $744 \pm 193$ & $1812 \pm 124$ \\
\hline & $1.2 \times 10^{7} \mathrm{CFU}$ & N.D. & $380 \pm 25^{* * *}$ & $1629 \pm 416^{* * *}$ & $1850 \pm 258$ \\
\hline & $2.4 \times 10^{7} \mathrm{CFU}$ & N.D. & $492 \pm 29^{* * *}$ & $1580 \pm 503^{* * *}$ & $1661 \pm 278$ \\
\hline & $4.8 \times 10^{7} \mathrm{CFU}$ & N.D. & $\begin{array}{c}498 \pm 35^{* * *} \\
(F=1093)\end{array}$ & $\begin{array}{c}1651 \pm 708^{* * *} \\
(F=20.82)\end{array}$ & $\begin{array}{c}1490 \pm 170^{* * *} \\
(F=5.044)\end{array}$ \\
\hline \multicolumn{6}{|l|}{ Treatment 2 (24 hours) } \\
\hline \multirow[t]{4}{*}{ GLe alone } & $0 \mu \mathrm{g} / \mathrm{mL}^{\dagger}$ & $6 \pm 11$ & $57 \pm 24$ & $645 \pm 102$ & $1765 \pm 115$ \\
\hline & $40 \mu \mathrm{g} / \mathrm{mL}$ & $7 \pm 13$ & $331 \pm 37^{* * *}$ & $1581 \pm 367^{* * *}$ & $1254 \pm 101^{* * *}$ \\
\hline & $80 \mu \mathrm{g} / \mathrm{mL}$ & $10 \pm 19$ & $822 \pm 35^{* * *}$ & $2430 \pm 397^{* * *}$ & $740 \pm 71^{* * *}$ \\
\hline & $100 \mu \mathrm{g} / \mathrm{mL}$ & $\begin{array}{c}22 \pm 29 \\
(F=1.238)\end{array}$ & $\begin{array}{c}1343 \pm 130^{* * *} \\
(F=541.6)\end{array}$ & $\begin{array}{c}3091 \pm 672^{* * *} \\
(F=105.2)\end{array}$ & $\begin{array}{c}671 \pm 62^{* * *} \\
(F=280.6)\end{array}$ \\
\hline \multicolumn{6}{|l|}{ Treatment 3 ( 24 hours) } \\
\hline $\mathrm{BCG}+\mathrm{GLe}$ & $0 \mu \mathrm{g} / \mathrm{mL}^{\dagger}$ & N.D. & $413 \pm 202$ & $1835 \pm 808$ & $1789 \pm 308$ \\
\hline \multirow[t]{3}{*}{$1.2 \times 10^{7} \mathrm{CFU}$} & $40 \mu \mathrm{g} / \mathrm{mL}$ & N.D. & $752 \pm 39^{*}$ & $1733 \pm 469$ & $520 \pm 137^{* * *}$ \\
\hline & $80 \mu \mathrm{g} / \mathrm{mL}$ & N.D. & $754 \pm 83^{* * *}$ & $1504 \pm 328$ & $818 \pm 169^{* * *}$ \\
\hline & $100 \mu \mathrm{g} / \mathrm{mL}$ & N.D. & $\begin{array}{c}891 \pm 40^{* * *} \\
(F=11.12)\end{array}$ & $\begin{array}{l}1436 \pm 338 \\
(F=1.628)\end{array}$ & $\begin{array}{c}542 \pm 101^{* * *} \\
(F=42.89)\end{array}$ \\
\hline \multicolumn{6}{|l|}{ Treatment 4 (48 hours) } \\
\hline 24-hr GLe $\rightarrow$ 24-hr BCG & $0 \mu \mathrm{g} / \mathrm{mL}^{\dagger}$ & N.D. & $640 \pm 44$ & $2256 \pm 830$ & $3471 \pm 338$ \\
\hline \multirow{3}{*}{$1.2 \times 10^{7} \mathrm{CFU}$} & $40 \mu \mathrm{g} / \mathrm{mL}$ & N.D. & $270 \pm 27^{* * *}$ & $874 \pm 68^{* * *}$ & $962 \pm 107^{* * *}$ \\
\hline & $80 \mu \mathrm{g} / \mathrm{mL}$ & N.D. & $164 \pm 31^{* * *}$ & $524 \pm 243^{* * *}$ & $89 \pm 103^{* * *}$ \\
\hline & $100 \mu \mathrm{g} / \mathrm{mL}$ & N.D. & $\begin{array}{c}161 \pm 20^{* * *} \\
(F=827.2)\end{array}$ & $\begin{array}{c}634 \pm 206^{* * *} \\
(F=43.45)\end{array}$ & $\begin{array}{c}\text { N.D. }{ }^{* * *} \\
(F=269.3)\end{array}$ \\
\hline
\end{tabular}

The secretion of cytokines detectable in the conditioned media collected from the cells treated with different treatment schedules (N.D.: non-detectable; $\left.{ }^{*} P<0.05 ;{ }^{* * *} P<0.001\right)$. Statistical significances of parameters in each treatment schedule group were compared with corresponding control ${ }^{\dagger}$. Controls for treatment 1 and 2 were not identical, because the 0 CFU BCG in treatment 1 contains 33\% (v/v) BCG diluents, whereas the $0 \mu \mathrm{g} / \mathrm{mL}$ GLe in treatment 2 contains $0.1 \%(\mathrm{v} / \mathrm{v})$ ethanol. Additionally, the diluting effect of BCG diluents in the complete media was suspected to be the cause of non-detectable level of IL-2 in treatment 1, as compared with the trace level produced in the GLe control in treatment 2. However, the secretion of other cytokines (IL-6, IL-8 and MCP-1 were not in trace amounts) was seemed to be not being affected by such dilution. $F$ value of each treatment group was determined by one-way ANOVA, and Dunnett's post test was followed to determine $P$ values.

malignant, since patients are apparently cured with complete tumor foci clearance. Herein, BCG has only demonstrated at the lowest tested concentration at $1.2 \times 10^{7} \mathrm{CFU}$ a small reduction (about 15\%) in viable cell number. Such cytotoxic effects were not observed at higher concentrations tested, in which the mechanism is uncertain. Action of BCG is believed to be relied on the inflammatory response upon urothelial internalization [18]. Subsequently, the expression of TNF-related apoptosis-inducing ligand (TRAIL/Apo2L) and Fas/CD95 ligand (FasL) was stimulated on infiltrated immune cells and Fas expression on tumor cells, resulting in tumor necrosis factor-alpha (TNF- $\alpha$-) induced apoptosis $[4,19]$. It was also supported by the findings that voided urine samples from TCC patients receiving BCG therapy were cytotoxic to RT-4 bladder cancer cells [4]. High levels of functional soluble forms of TRAIL were detected in such specimens [20]. In the present study, neither TNF- $\alpha$ nor IFN$\gamma$ was stimulated by the BCG treatment at the measured time point. However, FlowCytomix results revealed that IL6 and IL- 8 secretions were induced in the culture media of BCG-treated HUC-PC cells. IL-6 has evidenced for the ability in inducing interferon-gamma (IFN- $\gamma$ ), which is believed as a late responsive T-helper type 1 cytokine to stimulate the TRAIL expression, thus responsible for successful BCG prophylaxis [21,22]. Thus, current findings speculate the delay of BCG cytotoxicity was due to the process of BCG internalization and subsequent immune response, which requiring further elucidation.

On the other hand, activities of GLe have shown to be a fast action that early apoptotic events with positive annexin$\mathrm{V}$ uptake were initiated at 3 hours during the 24-hour incubation course [11]. Consistent with the reported G2M phase cell arrest in HUC-PC and other TCC cell lines [10], results herein have further demonstrated the progressing cytotoxicity and driven the complete adverse cell clearance several days after treatment retraction, especially at dose $80 \mu \mathrm{g} / \mathrm{mL}$ or above. However, before the GLe cytotoxicity can be translated into clinical applications, the specificity of such cytotoxicity to carcinoma or adverse cells must be defined. In preliminary studies, GLe has shown to exert mild 
cytotoxicity to normal uroepithelial (HUC-1) cells, but in a lesser extent than in the HUC-PC cells (data not shown), which suggests the selectivity on premalignant instead of normal urothelium. This is also consistent with the selective cytotoxic effects of G. lucidum on cancer cell lines versus normal cells in origins instead of urinary bladder [23]. Besides the antitumor activities, the administration of $G$. ludicum has increased the lifespan in tumor-bearing mice of mammary cancer and did not display any toxicity [24]. In human in vivo, G. lucidum did not cause toxic effects to harm the liver and kidneys of patients with breast cancer receiving conventional endocrine therapy, whereas the well-being and cancer-related fatigue were also improved [25].

Similar to the BCG activities, GLe was also shown to stimulate IL- 6 and IL- 8 in the HUC-PC cells. Induction of IL-6 secretion by other G. lucidum fractions has been reported from various cell types including human $\mathrm{T}$ cells and mouse splenocytes [26, 27]. The GLe-mediated IL-8 secretion was also correlated with neutrophilic chemotaxis attraction that may facilitate the apoptotic cell clearance [12]. Given that nuclear factor $-\kappa \mathrm{B}(\mathrm{NF}-\kappa \mathrm{B})$ and activating protein1 (AP-1) are the main signaling pathways responsible for cytokines induction, the expression of IL- 6 mRNA in the Hu35E6E7 HUC cells was found to be exclusively triggered by BCG through the Toll-Like Receptor (TLR) signaling [28]. Whilst p50/65 NF- $\kappa \mathrm{B}$ activity was enhanced by GLe concurrent with the cytokine elevation in the HUC-PC cells [12]. Therefore, the next question we asked was whether GLe would interact with BCG to favor TCC prophylaxis and how.

Based on the hypothesis that BCG induces interferon(IFN-) medicated cytotoxicity for the residual cell clearance, a 50-60\% initial response rate was evidenced in resistant and relapsing patients after receiving the intravesical instillation of IFN- $\alpha 2$ B and reduced dose of BCG [29]. Antitumor activities on bladder cancer cells were demonstrated by the combination treatment of Maitake mushroom with IFN- $\alpha$ [30]. In the present study, the cytotoxic effects exhibited by GLe and BCG were shown to be additive when cells were coincubated with both drugs or preincubated with GLe first. Despite the fact that both GLe and BCG are immunologically active, in the HUC-PC cultures, pretreatment with GLe was shown to be suppressive on all BCG-mediated cytokines. However, IL6 induced by BCG was further stimulated when coincubated with GLe, while IL- 8 secretion was inhibited. Such results implied that the inactivation of cytokine induction did not compromise the overall cytotoxicity, suggesting that the BCG and GLe synergism was cytokine-independent. On the contrary, the reduction of cytokine could be explained by the dramatic cytotoxic effects that essential metabolisms are commonly ceased during cell termination [31]. This could be explained by the early priming of HUC-PC cells by GLe to undergo progressive apoptosis before BCG was added [11]. Coherently, the antineoplastic properties of BCG on TCC cell lines [32] and mice in vivo [33] were enhanced by a vascular endothelial growth factor (VEGF)—Sunitinib through apoptotic pathways. Besides the therapeutic efficacy, bacterial infection and subsequent inflammatory response following intravesical BCG instillation are also associated with the high side effect tolls [7]. Despite such adverse effects

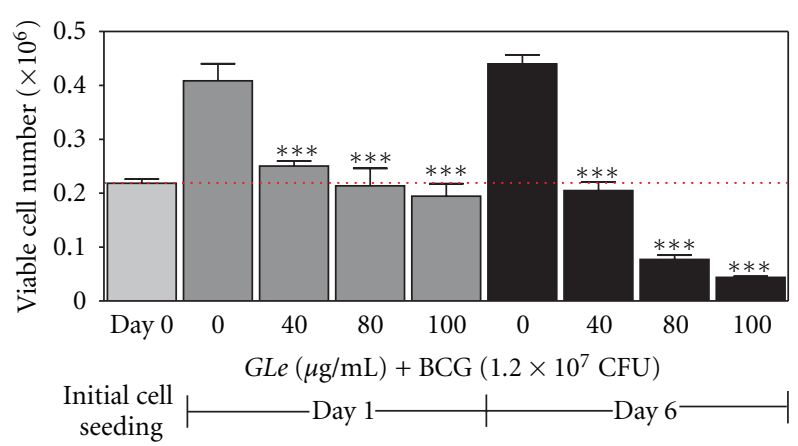

(a)

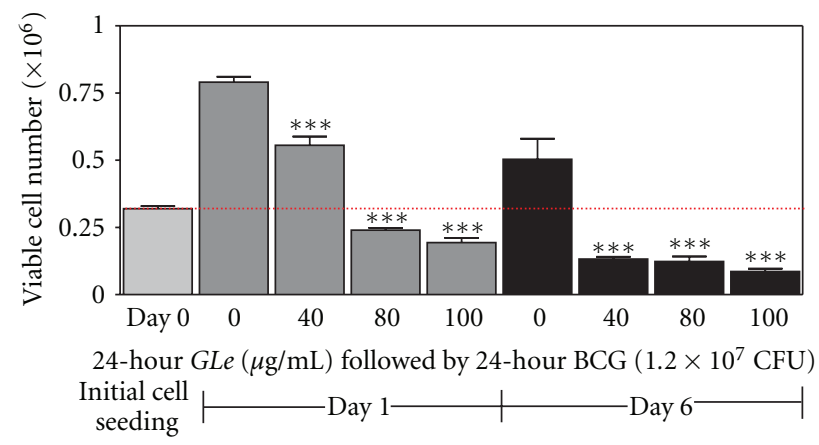

(b)

FIGURE 3: Showing the immediate and progressive cytotoxic effects exhibited by (a) coincubation of GLe with BCG on day 1 ( $F=$ $\left.14.14 ;{ }^{* * *} P<0.01\right)$ and day $6\left(F=217.6\right.$; $\left.{ }^{* * *} P<0.001\right)$, and (b) pretreatment of GLe followed by BCG on day 1 ( $F=169.8$; $\left.{ }^{* * *} P<0.001\right)$ and day $6(F=30.52 ; * * * P<0.01)$. For all test conditions, BCG concentration was fixed at $1.2 \times 10^{7} \mathrm{CFU}$ for testing different concentrations of GLe. Results were statistically compared with control at 0 GLe concentration but treated with BCG at $1.2 \times 10^{7} \mathrm{CFU}$, according to the corresponding schedule (i.e., day 1 versus day 1 and day 6 versus day 6). All experiments were performed in triplicate for reproducibility. $F$ value of each treatment group was determined by one-way ANOVA, and Dunnett's posttest was followed to determine $P$ values.

are frequently mild cases, with the commonest cystitis, fever and chills are at least partially linked with the induction of endogenous inflammatory cytokines in the bladder [5]. The incubation of NF- $\kappa \mathrm{B}$, which is a transcriptional factor for many cytokines, including the IL- 6 induced by GLe on HUC-PC cells [12], was shown to bear antitumor effects in other bladder cancer cells [34]. Interstitial cystitis is a form of inflammatory hypersensitivity characterized with high levels IL-6 and histamine in patients' urine [35]. This imposes another question whether the regulatory roles of GLe on BCG-mediated IL-6 and IL-8 would reduce also the side effects of BCG? However, more in-depth investigations are required using animal models.

Furthermore, MCP-1 expression of the HUC-PC cultures were dose-dependently inhibited by the treatments of BCG or GLe alone, combination therapy of BCG and GLe, and GLe pretreatment followed by BCG. It has reported that MCP-1 levels produced in the urine have directly correlated with the bladder cancer stages and grades [36]. Contradictorily, MCP-1 was elevated in the serum and 


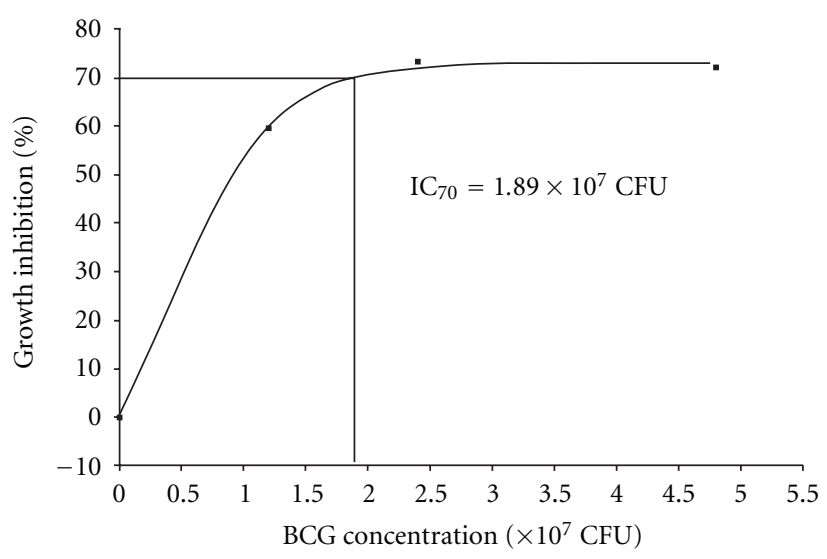

(a)

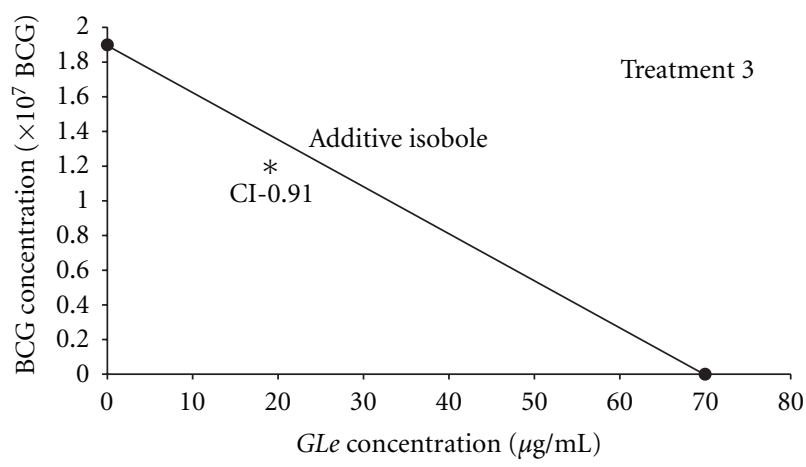

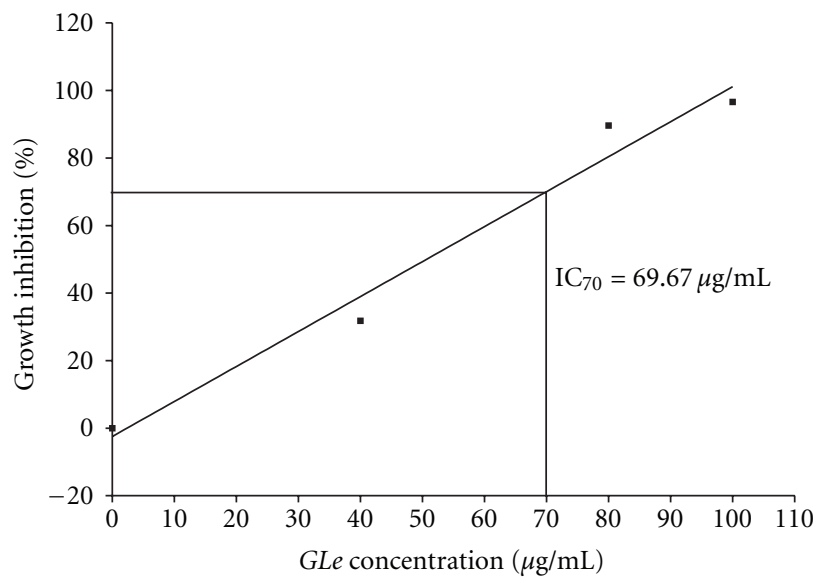

(b)

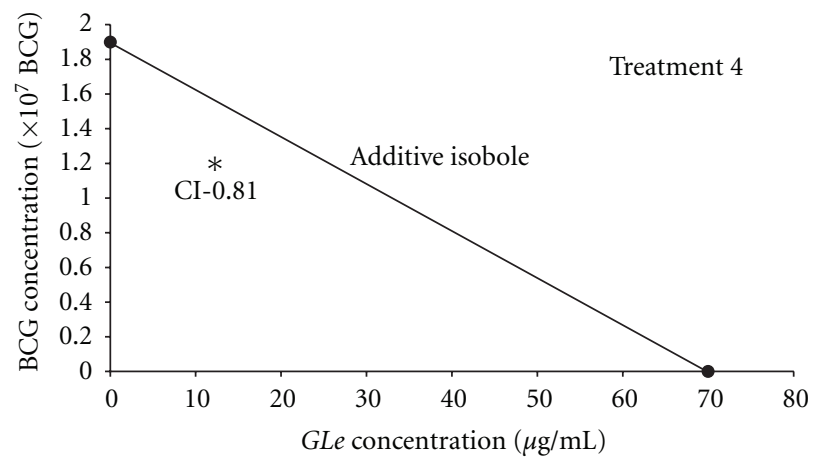

(c)

FIGURE 4: Dose-response curves of (a) BCG and (b) GLe generated by nonlinear regression, in order to define the sinGLe-agent effects as $\mathrm{IC}_{70}$. On day 6 following drug cessation, and since only selected BCG concentrations were tested, the BCG cytotoxicity showed ranged from $60-73 \%$ when compared with the solvent control (i.e., BCG 0 CFU on day 6). In order to minimize the error, IC 70 was used for isobologram analysis. Isobolograms (c) were plotted for treatments 3 and 4 with the $\mathrm{IC}_{70}$ determined for BCG and GLe. Concentrations of GLe and BCG were reflected on $x$ - and $y$-axes, respectively. Area below the additive isobole (the line joining $\mathrm{IC}_{70, \mathrm{BCG}}$ and $\mathrm{IC}_{70, G L e}$ ) indicates synergistic interaction. Combination indices $(\mathrm{CI})$ were calculated as $\mathrm{CI}=\mathrm{C}_{\mathrm{BCG}, 70} / \mathrm{IC}_{70, \mathrm{BCG}}+\mathrm{C}_{G L e, 70} / \mathrm{IC}_{70, G L e}$ according to Zhao et al. [14]. Specifically, $\mathrm{C}_{\mathrm{BCG}, 70}$ and $\mathrm{C}_{G L e, 70}$ are the concentrations of BCG and GLe used in treatments 3 and 4 to achieve $70 \%$ drug effect. IC $\mathrm{C}_{70, \mathrm{BCG}}$ (fixed as $1.2 \times 10^{7} \mathrm{CFU}$ for all experiments) and $\mathrm{IC}_{70, G L e}(19.12 \mu \mathrm{g} / \mathrm{mL}$ in treatment $3 ; 12.37 \mu \mathrm{g} / \mathrm{mL}$ in treatment 4$)$ are the concentrations for sinGLe agents to achieve the same effect.

bladder biopsy of bladder cancer patients following BCG immunotherapy [37]. The chemoattracting properties of MCP-1 were believed to be involved for tumor eradication; however, more recent studies have also demonstrated the roles of MCP-1 in angiogenesis and promoting tumor progression [11]. The pro- and antitumor effects of MCP1 remain controversial, but facts presented in this study have indicated that the premalignant HUC-PC cells were capable for MCP-1 production, whereas in response to GLe, the levels were significantly suppressed concurrently with the growth inhibition. The degree of MCP-1 suppression was clearly correlated with the cytotoxicity exerted by the combination therapy and pretreatment schedule.

\section{Conclusions}

Novel findings have demonstrated the possible synergistic interaction between BCG and GLe, in terms of adverse
TCC cells elimination. Both BCG and GLe were immunological active in the HUC-PC cells. However, the BCGprovoked cytokines were inhibited when the cells were first preincubated with GLe, proposing the potential implications in reducing the inflammatory-related toxicity of BCG and warranted more in-depth elucidation.

\section{Conflict of Interests}

There is no financial conflicts other relationship on needed to be disclosed in this submission.

\section{Acknowledgments}

This project was supported by the Research Committee of the Hong Kong Polytechnic University for the postgraduate scholarship (RGH8) and Sir Edward Youde Memorial Fellowship awarded to Dr. John Yuen. The authors are grateful 
to Dr. J. Y. Rao (UCLA Medical Center, USA) for providing the HUC-PC cell line and professional advice.

\section{References}

[1] R. Simon, E. Eltze, K. L. Schäfer et al., "Cytogenetic analysis of multifocal bladder cancer supports a monoclonal origin and intraepithelial spread of tumor cells," Cancer Research, vol. 61, no. 1, pp. 355-362, 2001.

[2] I. Sakai, H. Miyake, K. I. Harada, I. Hara, T. A. Inoue, and M. Fujisawa, "Analysis of factors predicting intravesical recurrence of superficial transitional cell carcinoma of the bladder without concomitant carcinoma in situ," International Journal of Urology, vol. 13, no. 11, pp. 1389-1392, 2006.

[3] J. J. Patard, F. Saint, F. Velotti, C. C. Abbou, and D. K. Chopin, "Immune response following intravesical bacillus CalmetteGuérin instillations in superficial madder cancer: a review," Urological Research, vol. 26, no. 3, pp. 155-159, 1998.

[4] A. T. Ludwig, J. M. Moore, Y. Luo et al., "Tumor necrosis factor-related apoptosis-inducing ligand: a novel mechanism for bacillus Calmette-Guérin-induced antitumor activity," Cancer Research, vol. 64, no. 10, pp. 3386-3390, 2004.

[5] D. L. Paterson and A. Patel, "Bacillus Calmette-Guérin (BCG) immunotherapy for bladder cancer: review of complications and their treatment," Australian and New Zealand Journal of Surgery, vol. 68, no. 5, pp. 340-344, 1998.

[6] E. C. de Boer, W. H. de Jong, P. A. Steerenberg et al., "Induction of urinary interleukin-1 (IL-1), IL-2, IL-6, and tumour necrosis factor during intravesical immunotherapy with bacillus Calmette-Guérin in superficial bladder cancer," Cancer Immunology Immunotherapy, vol. 34, no. 5, pp. 306312, 1992.

[7] R. J. Sylvester, A. P. M. van der Meijden, W. Oosterlinck, W. Hoeltl, and A. V. Bono, "The side effects of bacillus CalmetteGuérin in the treatment of Ta T1 bladder cancer do not predict its efficacy: results from a European organisation for research and treatment of cancer genito-urinary group phase III trial," European Urology, vol. 44, no. 4, pp. 423-428, 2003.

[8] E. A. Bookland, S. Swaminathan, R. Oyasu, K. W. Gilchrist, M. Lindstrom, and C. A. Reznikoff, "Tumorigenic transformation and neoplastic progression of human uroepithelial: cells after exposure in vitro to 4-aminobiphenyl or its metabolites," Cancer Research, vol. 52, no. 6, pp. 1606-1614, 1992.

[9] J. W. M. Yuen and M. D. I. Gohel, "Anticancer effects of Ganoderma lucidum: a review of scientific evidence," Nutrition and Cancer, vol. 53, no. 1, pp. 11-17, 2005.

[10] Q. Y. Lu, Y. S. Jin, Q. Zhang et al., "Ganoderma lucidum extracts inhibit growth and induce actin polymerization in bladder cancer cells in vitro," Cancer Letters, vol. 216, no. 1, pp. 9-20, 2004.

[11] J. W. M. Yuen, M. D. I. Gohel, and D. W. T. Au, "Telomerase-associated apoptotic events by mushroom Ganoderma lucidum on premalignant human urothelial cells," Nutrition and Cancer, vol. 60, no. 1, pp. 109-119, 2008.

[12] J. W. M. Yuen, M. D. I. Gohel, and C. F. Ng, "The differential immunological activities of Ganoderma lucidum on human pre-cancerous uroepithelial cells," Journal of Ethnopharmacology, vol. 135, no. 3, pp. 711-718, 2011.

[13] J. W. M. Yuen and M. D. I. Gohel, "The dual roles of Ganoderma antioxidants on urothelial cell DNA under carcinogenic attack," Journal of Ethnopharmacology, vol. 118, no. 2, pp. 324330, 2008.
[14] L. Zhao, M. G. Wientjes, and J. L. S. Au, "Evaluation of combination chemotherapy: integration of nonlinear regression, curve shift, isobologram, and combination index analyses," Clinical Cancer Research, vol. 10, no. 23, pp. 7994-8004, 2004.

[15] Y. Zhang, H. E. Khoo, and K. Esuvaranathan, "Effects of bacillus Calmette-Guérin and interferon- $\alpha-2 \mathrm{~B}$ on human bladder cancer in vitro," International Journal of Cancer, vol. 71, no. 5, pp. 851-857, 1997.

[16] M. C. Filion, P. Lépicier, A. Morales, and N. C. Phillips, "Mycobacterium phlei cell wall complex directly induces apoptosis in human bladder cancer cells," British Journal of Cancer, vol. 79, no. 2, pp. 229-235, 1999.

[17] A. Sasaki, S. Kudoh, K. Mori, N. Takahashi, and T. Suzuki, "Are BCG effects against urinary bladder carcinoma cell line T24 correlated with apoptosis in vitro?" Urologia Internationalis, vol. 59, no. 3, pp. 142-148, 1997.

[18] R. F. M. Bevers, K. H. Kurth, and D. H. J. Schamhart, "Role of urothelial cells in BCG immunotherapy for superficial bladder cancer," British Journal of Cancer, vol. 91, no. 4, pp. 607-612, 2004.

[19] M. Mehmut, K. Takeda, M. Abe et al., "Fas ligand and TNFrelated apoptosis-inducing ligand induction on infiltrating lymphocytes in bladder carcinoma by bacillus CalmetteGuérin treatment," Urologia Internationalis, vol. 75, no. 1, pp. 80-87, 2005.

[20] T. J. Kemp, A. T. Ludwig, J. K. Earel et al., "Neutrophil stimulation with Mycobacterium bovis bacillus CalmetteGuérin (BCG) results in the release of functional soluble TRAIL/Apo-2L," Blood, vol. 106, no. 10, pp. 3474-3482, 2005.

[21] Y. Luo, X. Chen, and M. A. O'Donnell, "Role of Th1 and Th2 cytokines in BCG-induced IFN- $\gamma$ production: cytokine promotion and simulation of BCG effect," Cytokine, vol. 21, no. 1, pp. 17-26, 2003.

[22] K. G. Nepple, H. A. Aubert, M. R. Braasch, and M. A. O'Donnell, "Combination of BCG and interferon intravesical immunotherapy: an update," World Journal of Urology, vol. 27, no. 3, pp. 343-346, 2009.

[23] Y. H. Gu and M. A. Belury, "Selective induction of apoptosis in murine skin carcinoma cells (CH72) by an ethanol extract of Lentinula edodes," Cancer Letters, vol. 220, no. 1, pp. 21-28, 2005.

[24] Y. Nonaka, H. Shibata, M. Nakai et al., "Anti-tumor activities of the antlered form of Ganoderma lucidum in allogeneic and syngeneic tumor-bearing mice," Bioscience, Biotechnology and Biochemistry, vol. 70, no. 9, pp. 2028-2034, 2006.

[25] H. Zhao, Q. Zhang, L. Zhao, X. Huang, J. Wang, and X. Kang, "Spore powder of Ganoderma lucidum improves cancerrelated fatigue in breast cancer patients undergoing endocrine therapy: a pilot clinical trial," Evidence-Based Complementary and Alternative Medicine, vol. 2012, Article ID 809614, 8 pages, 2012.

[26] W. T. Chung, S. H. Lee, J. D. Kim et al., "Effect of mycelial culture broth of Ganoderma lucidum on the growth characteristics of human cell lines," Journal of Bioscience and Bioengineering, vol. 92, no. 6, pp. 550-555, 2001.

[27] H. S. Chen, Y. F. Tsai, S. Lin et al., "Studies on the immuno-modulating and anti-tumor activities of Ganoderma lucidum (Reishi) polysaccharides," Bioorganic and Medicinal Chemistry, vol. 12, no. 21, pp. 5595-5601, 2004.

[28] J. Miyazaki, K. Kawai, T. Oikawa et al., "Uroepithelial cells can directly respond to Mycobacterium bovis bacillus CalmetteGuérin through Toll-like receptor signalling," British Journal of Urology International, vol. 97, no. 4, pp. 860-864, 2006. 
[29] M. A. O’Donnell, J. Krohn, and W. C. DeWolf, "Salvage intravesical therapy with interferon- $\alpha 2 \mathrm{~B}$ plus low dose bacillus Calmette-Guérin is effective in patients with superficial bladder cancer in whom bacillus Calmette-Guérin alone previously failed," Journal of Urology, vol. 166, no. 4, pp. 1300$1304,2001$.

[30] B. Louie, S. Rajamahanty, J. Won, M. Choudhury, and S. Konno, "Synergistic potentiation of interferon activity with maitake mushroom d-fraction on bladder cancer cells," British Journal of Urology International, vol. 105, no. 7, pp. 1011-1015, 2010.

[31] R. G. Jones and C. B. Thompson, "Tumor suppressors and cell metabolism: a recipe for cancer growth," Genes and Development, vol. 23, no. 5, pp. 537-548, 2009.

[32] S. Y. Ping, C. L. Wu, and D. S. Yu, "Sunitinib can enhance BCG mediatedcytotoxicity to transitional cell carcinoma through apoptosis pathway," Urologic Oncology: Seminars and Original Investigations. In press.

[33] D. S. Yu, S. Y. Ping, C. L. Wu, H. I. Chen, S. Y. Chang, and C. P. Ma, "The feasibility of BCG and sunitinib combination therapy for transitional cell carcinoma," Urological Science, vol. 22, no. 1, pp. 19-27, 2011.

[34] Y. Horiguchi, K. Kuroda, J. Nakashima, M. Murai, and K. Umezawa, "Antitumor effect of a novel nuclear factor- $\kappa \mathrm{B}$ activation inhibitor in bladder cancer cells," Expert Review of Anticancer Therapy, vol. 3, no. 6, pp. 793-798, 2003.

[35] L. M. Lamale, S. K. Lutgendorf, M. B. Zimmerman, and K. J. Kreder, "Interleukin-6, histamine, and methylhistamine as diagnostic markers for interstitial cystitis," Urology, vol. 68, no. 4, pp. 702-706, 2006.

[36] B. Amann, F. G. E. Perabo, A. Wirger, H. Hugenschmidt, and W. Schultze-Seemann, "Urinary levels of monocyte chemoattractant protein-1 correlate with tumour stage and grade in patients with bladder cancer," British Journal of Urology, vol. 82, no. 1, pp. 118-121, 1998.

[37] M. Reale, R. Intorno, R. Tenaglia et al., "Production of MCP-1 and RANTES in bladder cancer patients after bacillus Calmette-Guérin immunotherapy," Cancer Immunology, Immunotherapy, vol. 51, no. 2, pp. 91-98, 2002. 


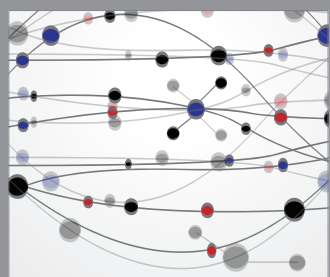

The Scientific World Journal
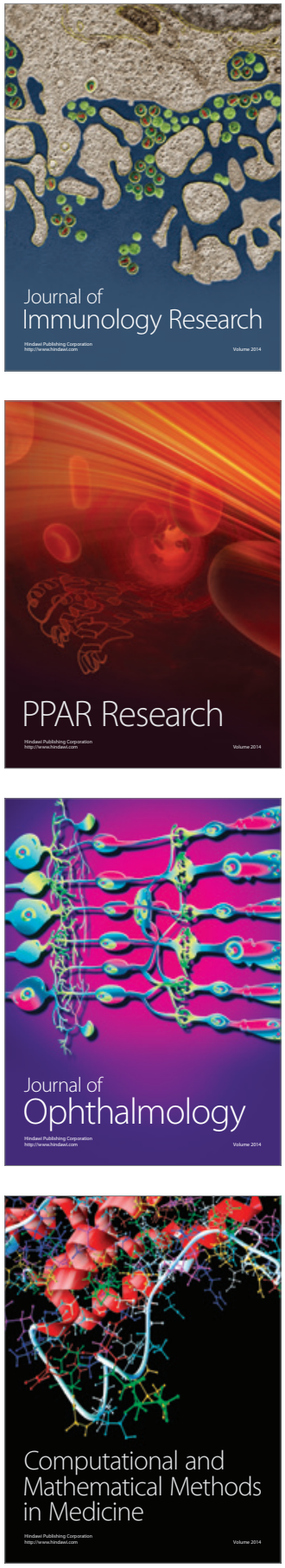

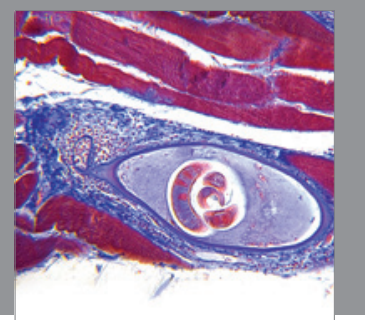

Gastroenterology

Research and Practice
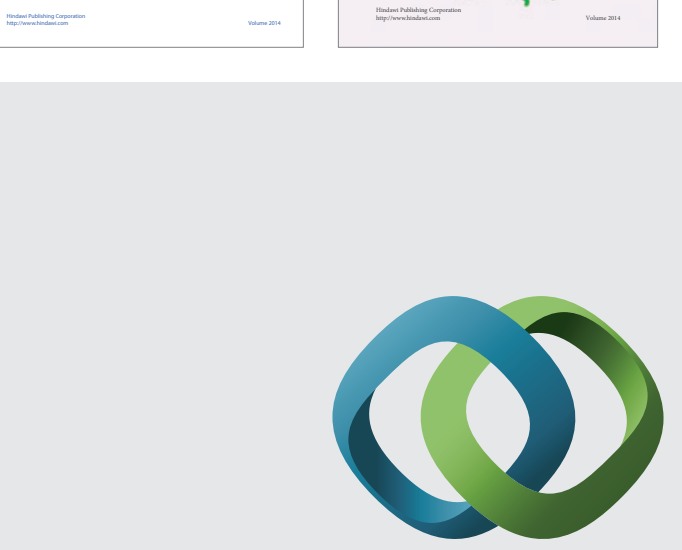

\section{Hindawi}

Submit your manuscripts at

http://www.hindawi.com
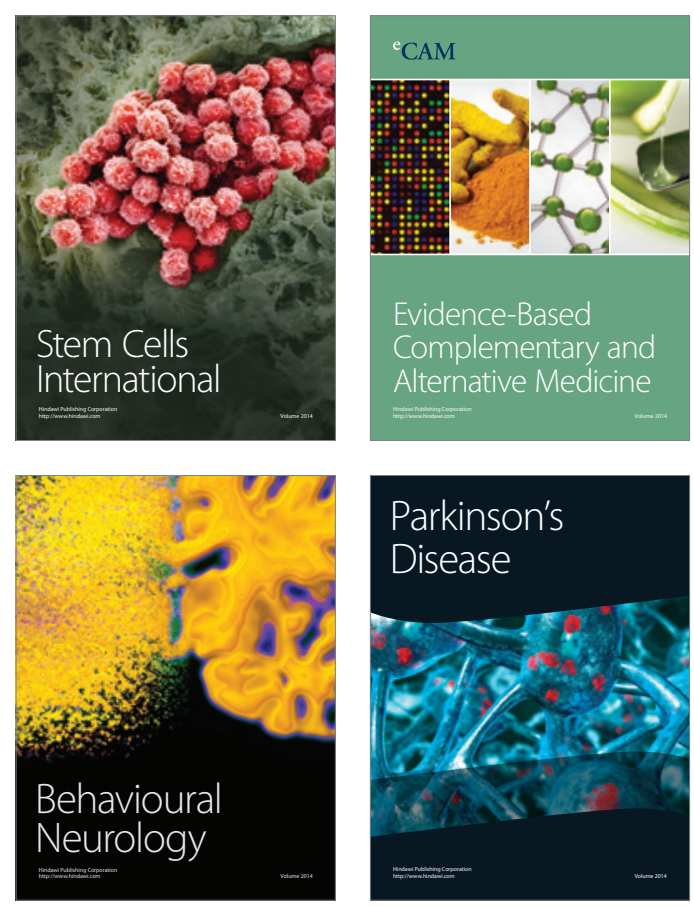

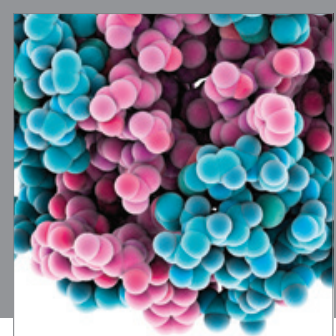

Journal of
Diabetes Research

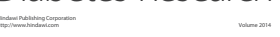

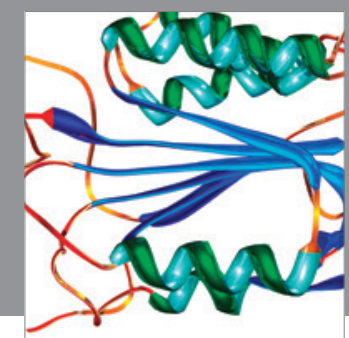

Disease Markers
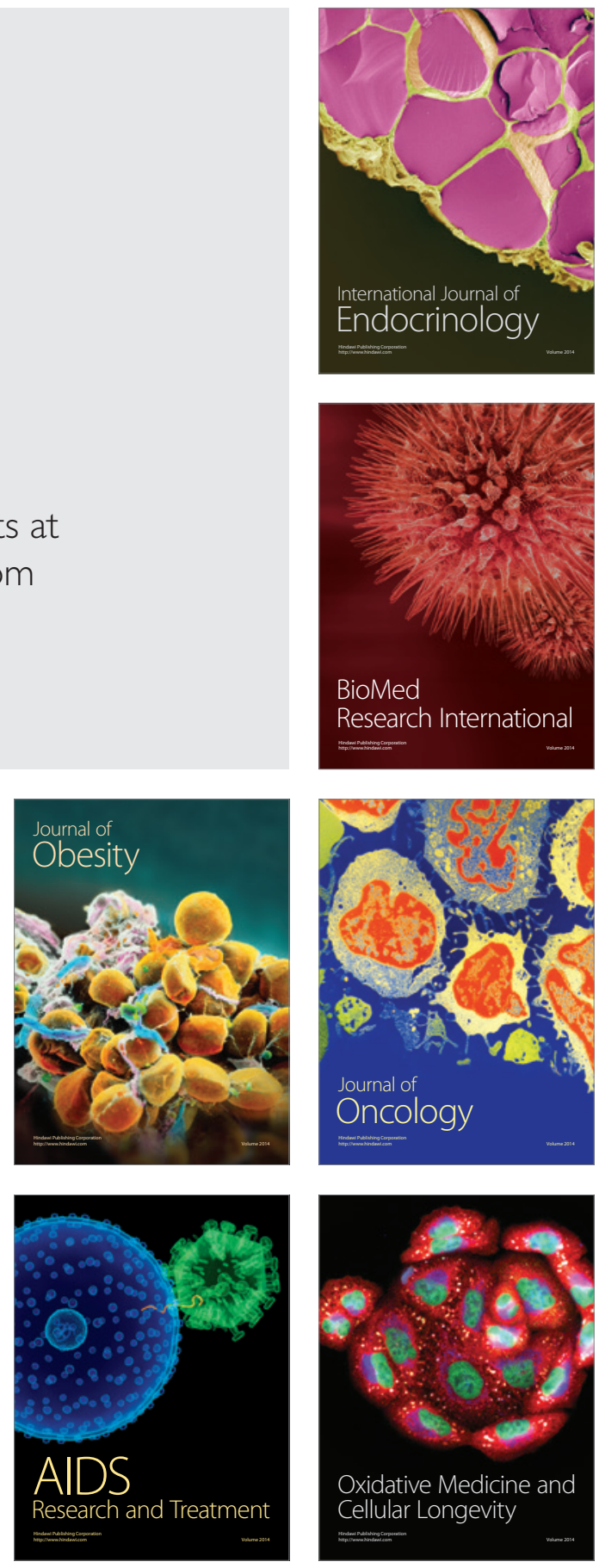